patients, whereas, $3(2.2 \%)$ patients underwent pelvic and para-aortic lymph node dissection. Median number of dissected lymph nodes was 11 (interquartile range: 5.7-21.2). Among patients who underwent lymph node dissection, 14 (16.3\%) patients showed lymph node involvement. Stage I, II and III were recorded in 97(71.9\%), 13 (9.6\%), and 25 (18.5\%) patients respectively. We did not observe lymphedema in our study participants. Other complications related to lymph node dissection were low grade and were not associated with age, BMI, extent of lymph node dissection, total number of dissected lymph nodes, lymph node involvement and disease stage.

Conclusion Complications related to lymph node dissection including lymphedema are rare after $\mathrm{TAH}+\mathrm{BSO}$ for endometrial cancer and the extent of lymph node dissection or disease stage is not associated with higher risk of such complications.

\section{CLINIC-PATHOLOGICAL FEATURES OF MIXED ENDOMETRIAL CARCINOMA; EVALUATION OF 29 PATIENTS IN A SINGLE TERTIARY CENTER}

Mete Sucu, Omer Faruk Geckil, Umran Kucukgoz Gulec, Ahmetbaris Guzel, Ghanim Khatib, Mehmet Ali Vardar. Cukurova University Faculty of Medicine; Obstetrics and Gynecology

\subsection{6/ijgc-2020-ESG0.217}

Introduction/Background Mixed endometrial carcinoma (MEC) refers to a tumor that is comprised of two or more distinct histotypes. Each component histotype by definition has to represent more than $5 \%$ of the tumor. Although it is relatively rare, both diagnosis and management can be troublesome. Molecular and histopathologic features have become important in the identifcation and more importantly the precise management of the MEC's.

In our study, we aimed to evaluate the clinical and pathological characteristics of the MEC

Methodology The clinical and pathological records of the 29 MEC patients who were operated on and regularly followed up in the clinic between January 2000-December 2019 were reviewed. Clinical features, operation characteristics, pathological findings, myometrial invasion degree (MI), lymph node involvement (LNI), lymphovascular space invasion (LVSI), adjuvant therapies, and follow-up data of the patients and their effects on survival were investigated.

Results During the study period, 29 out of 1110 patients with endometrial cancer had MEC (2.6\%). Eighteen of the cases had endometrioid + serous, 7 had endometrioid + clear, 3 had endometrioid + serous, and 1 had clear + serous histopathology. The mean age of the patients was $63.2 \pm 12.1$. Laparoscopic surgery was performed in 8 of the cases $(27.6 \%)$. Sixteen of the cases were in stage $1(55.1 \%), 4$ were in stage $2(13.8 \%)$, and 9 were in stage $3(31 \%)$. LVSI was positive in 17 cases (58.6\%). LNI was detected in 7 cases (24.1\%). Approximately 80 percent of cases received adjuvant therapy. While $80 \%$ of the cases received chemotherapy, this rate was $55 \%$ for radiotherapy.

Conclusion MECs are tumors that can be difficult to diagnose and manage. In addition to histopathological features, revealing and evaluating their molecular properties will help us to better understand this group of tumors.

Disclosures None

\section{Miscellaneous}

\section{IMPACT OF THE COVID PANDEMIC ON GYNAECOLOGICAL CANCER SURGERY - RESULTS FROM THE COVIDSURG GYNAECOLOGICAL CANCER INTERNATIONAL STUDY}

${ }^{1}$ Sudha S Sundar, ${ }^{1}$ Elaine Leung, ${ }^{2}$ Tabassum Khan, ${ }^{1}$ James Glasbey, ${ }^{3}$ Nadeem Abu-Rustum, ${ }^{4}$ Luis M Chiva, ${ }^{5}$ Anna Fagotti, ${ }^{6}$ Keiichi Fujiwara, ${ }^{7}$ Rahel Ghebre, ${ }^{8}$ Murat Gultekin, ${ }^{9}$ Thomas Konney, ${ }^{10}$ Joseph Ng, ${ }^{11}$ Rene Pareja, ${ }^{12}$ Rajkumar Kottayasamy Seenivasagam, ${ }^{13}$ Jalid Sehouli, ${ }^{14}$ Ts Shylasree, ${ }^{1}$ Aneel Bhangu, ${ }^{15}$ Christiana Fotopoulou, ${ }^{16}$ Covidsurg Cancer Collaborators. 'University of Birmingham; Institute of Cancer and Genomic Sciences; University of Birmingham; ' University of Birmingham; Nihr Global Health Research Unit on Global Surgery; ${ }^{3}$ Memorial Sloan Kettering Cancer Center; ${ }^{4}$ Clínica Universidad de Navarra; ${ }^{5}$ Catholic University of the Sacred Heart; Fondazione Policlinico A. Gemelli; ${ }^{6}$ Saitama Medical University; International Medical Center; ' University of Minnesota; Department of Obstetrics, Gynaecology and Women's Health; ${ }^{8}$ Hacettepe University Faculty of Medicine; Department of Obstetrics and Gynaecology; ${ }^{9}$ Kwame Nkrumah University Of Science and Technology; Komfo Anokye Teaching Hospital; ${ }^{10}$ National University Cancer Institute; Division of Gynecologic Oncology; ${ }^{11}$ Clinica de Oncología Astorga; Gynaecologic Oncology; ${ }^{12}$ All India Institute of Medical Sciences; Obstetrics and Gynaecology; ${ }^{13}$ Charité Universitätsmedizin Berlin; Klinik für Gynäkologie; Charité Universitätsmedizin Berlin; ${ }^{14}$ Tata Memorial Hospital; Department of Gynecologic Oncology and Mdt; ${ }^{15} /$ mperial College London; ${ }^{16}$ Nihr Global Health Research Unit

\subsection{6/ijgc-2020-ESG0.218}

Introduction/Background Covid-19 has resulted in significant number of elective surgeries being delayed or cancelled worldwide with an estimated 28 million patients being affected. ${ }^{1}$

Studies show that perioperative Covid-19 infection has a high perioperative mortality of $23 \cdot 8 \%$. (2) Complications increase with any additional treatment burden such as cytotoxic chemotherapy, radiotherapy or immunotherapy. ${ }^{3}$ In an effort to reduce treatment related morbidity and mortality during the Covid-19 pandemic, many elective anticancer treatments have been postponed or modified. ${ }^{4}$

Methodology We investigated the impact of the Covid-19 pandemic on gynaecological cancer surgery in an international prospective multi-centre study. Participating centres entered consecutive patient's data into a customized electronic database for 12 weeks from the first COVID positive patient managed in their hospital between March and June 2020. Patients were eligible for enrollment if they were planned to undergo surgery during the study duration, regardless of COVID-19 status and whether they underwent surgery as recommended or not. Patients who did not undergo their planned surgery were followed up for 12 weeks to observe outcomes. Descriptive analysis of outcomes is presented.

$4490 / 4472(95 \%)$ patients received surgery; of these $17 \%$ $(n=758)$ experienced change or adaptation of surgery. The main impact was on surgical timing; $11 \%(n=483)$ experienced delay in surgery, 3\% $(n=119)$ a change in choice of operation, $10 \% \quad(\mathrm{n}=452)$ received surgery in alternative hospital.

Patients in this study had confirmed resolved COVID-19 prior to surgery in $0.95 \%(n=45)$ patients with an additional $0.34 \%(n=16)$ with probable resolved COVID-19 infection. A post-operative COVID-19 rate of $2.27 \%(n=25)$ and pulmonary complication rate of $1.8 \%(n=20)$ was found in the initial analysis of the Covidsurg cancer data, analysing outcomes for 1102 gynaecological cancer patients. The overall 30-day mortality rate in this cohort was $1.18 \%(n=13) .^{5}$

Conclusion The largest multi-centre analysis of gynaecological cancer surgery during the Covid-19 pandemic has 